\title{
Nailfold videocapillaroscopy in internal medicine
}

\author{
Paola Faggioli, Antonio Tamburello, Alba Sciascera, Adele Giulia Gilardi, Antonino Mazzone \\ Department of Internal Medicine, Hospital of Legnano, Legnano (MI), Italy
}

\begin{abstract}
Capillaroscopy is an actual inexpensive imaging technique, used to examine, non-invasively and safely, the morphology of nailfold dermal papillary capillaries. Many studies agree in the statement that the capillaroscopy is one of the gold standard methods for non-invasive examination of the microcirculation and it plays an important role in screening in Raynaud's phenomenon and in monitoring of systemic sclerosis and other rheumatologic diseases. There are also many reports on the possible use of nailfold capillaroscopy in the diagnosis and monitoring of many other diseases in internal medicine.
\end{abstract}

\section{Nailfold videocapillaroscopy}

\section{Short history}

Capillaroscopy is an old but actual inexpensive imaging technique, used to examine, non-invasively and safely, the morphology of nailfold dermal papillary capillaries. In the nailfold distal capillary row the dermal papillae run parallel to the surface of the nail, subsequently the capillaries of the distal row are visible in their whole length and appear as red, hairpinshaped loops. First Johan Christophorus Kolhaus, in 1663, had the idea to observe small vessels around the nails using a rudimentary microscope and about a hundred years later Giovanni Rasori (1766-1873), described the inextricable knot of capillary loops observed with a magnifying glass in inflamed conjunctives. After almost other hundred years, Maurice Raynaud (1834-1881), with his studies on local ischemic damage, validated and promoted capillaroscopy as a fundamental imaging technique for the study of microcirculation. ${ }^{1}$

Correspondence: Paola Faggioli, Department of Internal Medicine, Hospital of Legnano, via Giovanni Paolo II, 20025 Legnano (MI), Italy.

Tel.: +39.0331.449305 - Fax: +39.0331.449441.

E-mail: p.faggioli@libero.it

Key words: Nailfold videocapillaroscopy; internal medicine.

See online Appendix for additional Figures.

Received for publication: 6 October 2014.

Accepted for publication: 3 December 2014.

This work is licensed under a Creative Commons Attribution NonCommercial 3.0 License (CC BY-NC 3.0).

CCopyright P. Faggioli et al., 2015

Licensee PAGEPress, Italy

Italian Journal of Medicine 2015; 9:234-242

doi:10.4081/itjm.2015.548
In 1973, Maricq et al. ${ }^{2}$ published the first article in Arthritis and Rheumatism, describing the specific capillaroscopic patterns in systemic sclerosis (SSc), as well as the modification of the capillary blood flow during cold exposure, both in primary and secondary Raynaud's phenomenon (RP). ${ }^{2}$

In 2013 the power of the non-invasive nailfold videocapillaroscopy (NVC) technique, has been recognized for the early diagnosis of the scleroderma spectrum diseases, its predictivity and prognostic value, as well as its role as a tool for the therapeutic follow up. After more than 30 years of intensive investigation, capillaroscopy is now officially considered by the European League Against Rheumatism (EULAR) and American College of Rheumatology (ACR) guidelines as both an essential and mandatory diagnostic tool for the classification criteria of systemic sclerosis. ACR/EULAR stated in the 2013 guidelines for classification criteria of SSc: Capillaroscopy is now widely used, and considering the value of magnified nailfold visualization in the diagnosis and management of SSc, these new criteria may encourage acquisition of this skill by physicians caring for SSc patients. ${ }^{1}$

\section{Technique}

Using light microscopy capillaroscopy it is possible to evaluate the architecture of the skin capillaries. For this the nailfolds of the fingers, where the capillaries run parallel to the skin surface and can be evaluated in their entire architecture, are particularly suitable. $^{3}$

Capillaroscopy may be performed with a lens with low (e.g., 20) and with high magnification (e.g., 200). The instruments with low magnification allow a global and a panoramic evaluation of the entire nailfold area (wide-field capillaroscopy). These instruments allow a panoramic vision of the whole nailfold microvascular network. One example of an optical instrument 
with low magnification is the stereomicroscope (e.g., magnification $14 \mathrm{x}$ ).

Of note, with this technique the seminal descriptive papers concerning the scleroderma pattern were described by Maricq et al. in the last century (see below). Alternative choices are the dermatoscope and ophthalmoscope.

The video-capillaroscope not only allows low magnification, but also has the advantage of having sequential high magnifications (e.g., magnifications 100, 200 and 600x), which enable detailed observations of separate capillaries.

Many different techniques are adopted to perform NVC, with similar results and have also been exposed in a recent paper of Mazzotti et al., ${ }^{4}$ which compares conventional capillaroscopy, using the gold standard method (conventional stereomicroscope nailfold capillaroscopy), with polarized and non-polarized light contact dermoscopy and his conclusions are that both polarized and non-polarized dermoscopy are reliable methods for evaluation of naifold capillaroscopy in patients with SSc. They are easy to perform, with gold rates of accuracy and results that are comparable with traditional capillaroscopy. Another advantage of the video-capillaroscope is that it consists of an optical probe, which is moved to the finger of the patient and allows direct contact with the nailfold. This facilitates examination of patients with SSc and severe finger flexion contractures. ${ }^{5}$

Usually the exam is applied to the naifold area of the fingers, $8 / 10$ fingers and the focus cover about 1 $\mathrm{mm}$ of cutaneous area. In some cases capillaroscopy could be used to study other areas of the body, including the lips, tongue, mouth and conjunctival mucosa. ${ }^{6}$

In order to avoid bias in exam naifold microtraumas, onicophagia, smoking, nail, varnish should be avoided and the room temperature should be constant and over $21-22^{\circ} \mathrm{C}$ to reduce some possible RP attacks. On the other hand, feet nailfold capillaroscopy is not useful to detect scleroderma pattern for less sensibility to detect avascular areas, microhemorrhages and giant capillaries (topics of scleroderma pattern active), ${ }^{7}$ sometimes it is possible to detect a little difference in arterial peripheral disease (see personal data). A criticality to capillaroscopy was an intra-observer variability in the determination of capillary density under many different techniques; several subsequent studies showed the assessment of capillary density with different methods as computed videocapillaroscopy, stereo zoom microscopy or dermatoscopy showed good agreement in detecting scleroderma pattern. ${ }^{8}$

The examination includes the assessment of morpho-structural and functional parameters: transparency of the skin, venous plexus, capillary orientation, density, length, diameter, presence of neoangiogenesis, capillary morphology, micro-hemorrhages, character- istics of blood flow. Each of these parameters is evaluated according to a semi-quantitative score validated in a scale between 0 and 3 (alterations respectively in $33 \%, 33 \%-66 \%,>66 \%$ in NVC observation). ${ }^{9}$

\section{Indications to perform a capillaroscopy}

The main indication is investigation of a patient with RP. This way it is possible to distinguish a RF primitive from RF secondary to SSc, where pathognomonic changes on capillaroscopy are present. In addition, patients with RP as the only presenting symptom, but who will develop SSc in the future can be detected years before the appearance of the disease by a screening using the combination of capillaroscopy and serology autoimmunity.

\section{Normal pattern}

Under physiological conditions the normal pattern is characterized by: i) the orderly arrangement of the capillaries to comb; ii) density of 9-13 mm (maximum 3 per dermal papilla); iii) 6-9 micron diameter afferent branch, efferent branch 8-21 micron (>50 micron: megacapillaries); iv) length 200-500 $\mu$.

It should not be present, if not exceptionally, bleeding, increased angiogensis, megacapillaries, the presence of which already indicates a pathological pattern.

Based on this analysis we could depict three major normal morphologic capillaroscopic patterns: i) the normal pattern mainly with 2 to $5 \mathrm{U}$-shaped loops $/ \mathrm{mm}$ and $\leq 2$ tortuous loops $/ \mathrm{mm}$ (cluster 1); ii) the perfect normal pattern with $\geq 5 \mathrm{U}$-shaped loops $/ \mathrm{mm}$ (cluster 2 ); iii) the unusual normal with at least 1 meandering or bushy loop, or at least 1 microhemorrhage, or with $\mathrm{N} 4$ crossed loops/mm (clusters 3-4-5-6) ${ }^{10}$ (Figure 1).

Nagy et al. reported the frequency of abnormal capillaroscopy findings in 447 patients with collagen disorders and Raynaud's disease to be $87.5 \%$ in those

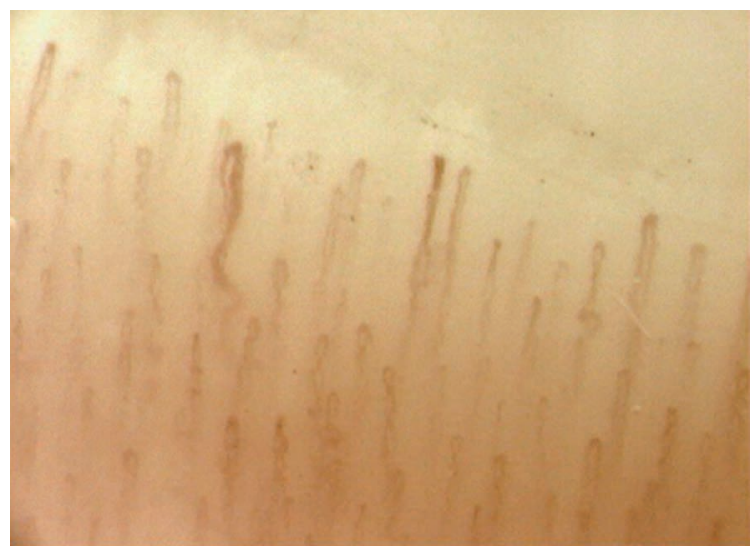

Figure 1. Capillaroscopic normal pattern. 
with scleroderma, $26.4 \%$ in those with dermatomyositis/polymyositis and $8.5 \%$ in those with systemic lupus erythematosus. ${ }^{11}$

\begin{abstract}
Abnormal patterns/scleroderma pattern
The interpretation of capillaroscopic examination involves the analysis of the parameters listed above and in particular the evaluation of orientation and distribution of the comb, the presence of megacapillaries, accumulations of hemosiderin, thrombosis, neoangiogenesis, presence of avascular areas. According to the different proportions of these parameters (giants capillaries, capillary loss, microhemorrhages, and ramifications) it was possible to establish a specific pattern for diseases related to the scleroderma spectrum syndrome for which the sensitivity and specificity is very high. Patognomonic capillaroscopic findings of scleroderma spectrum are: the presence of at least one capillary giant $(>70 \mu)$ or two megacapillaries $(>50 \mu)$, the presence of avascular areas $(500 \mu)$ or decreased capillary density, the presence of microhemorrhages, changes in neoangiogenesis, flou appearance of the capillary bed ${ }^{12}$ (Figure 2 ).
\end{abstract}

\section{Diagnostic role of capillaroscopy}

Capillaroscopy is of outstanding importance in the differential diagnosis of Raynaud's syndrome with a high negative predictive value in normal findings and high specificity of the SSc pattern for the presence of development of SSc. It is more important to stratify the risk of development RF-II o SSc secondary to RF. ${ }^{13}$

This algorithm approved also by the Rheumatologic Scientific Societies stresses the importance of early detection of capillaroscopic anomalies to improve the diagnosis of scleroderma syndromes, importance stressed also by VEDOSS classification for detection of very early SSc according to the European
Scleroderma Trials and Research group (EUSTAR)/ EULAR guidelines. ${ }^{14}$

\section{Role of capillaroscopy in classification criteria of systemic sclerosis}

After 2000 capillaroscopic markers of the scleroderma pattern were introduced in published classification criteria and in 2001 a role of capillaroscopy was established in differentiating a RPI from an RP due to $\mathrm{SSc}$, the presence of a scleroderma pattern on capillaroscopy and SSc-specific antibodies were stated to be sufficient to diagnose early (preclinical) SSc.

In 2001 capillaroscopic scleroderma pattern in association with RF, puffy swollen fingers turning into sclerodactily, positive anti-centromere autoantibodies (ACAs) and positive anti-topoisomerase antibodies are definite gold standard for the very early diagnosis of SSc (VEDOSS criteria).

In 2012 capillaroscopy has become one of the top items useful for classification of SSc. ${ }^{15}$

\section{Prognostic value}

Recently, prognostic indices based on capillaroscopy were created and applied exclusively in the diagnosis and confirmation of RP with the possibility of progression to scleroderma.

The main and validated are: i) PRINCE (prognostic index for nailfold capillaroscopic examination); ${ }^{16}$ ii) CSURI (capillaroscopic skin $u$ lcer $r$ isk index). ${ }^{15}$

The combination of the following 4 parameters: megacapillaries, microhemorrhages number of capillaries $/ \mathrm{mm}^{2}$, presence of ACA or Scl70, to which is assigned a score, permits to classify 3 risk classes (low, intermediate, high) indicative of the evolution in systemic sclerosis over 5 years.

PRINCE index is predictor of the occurrence of new digital ulcers or non-healing of open sores, in pa-

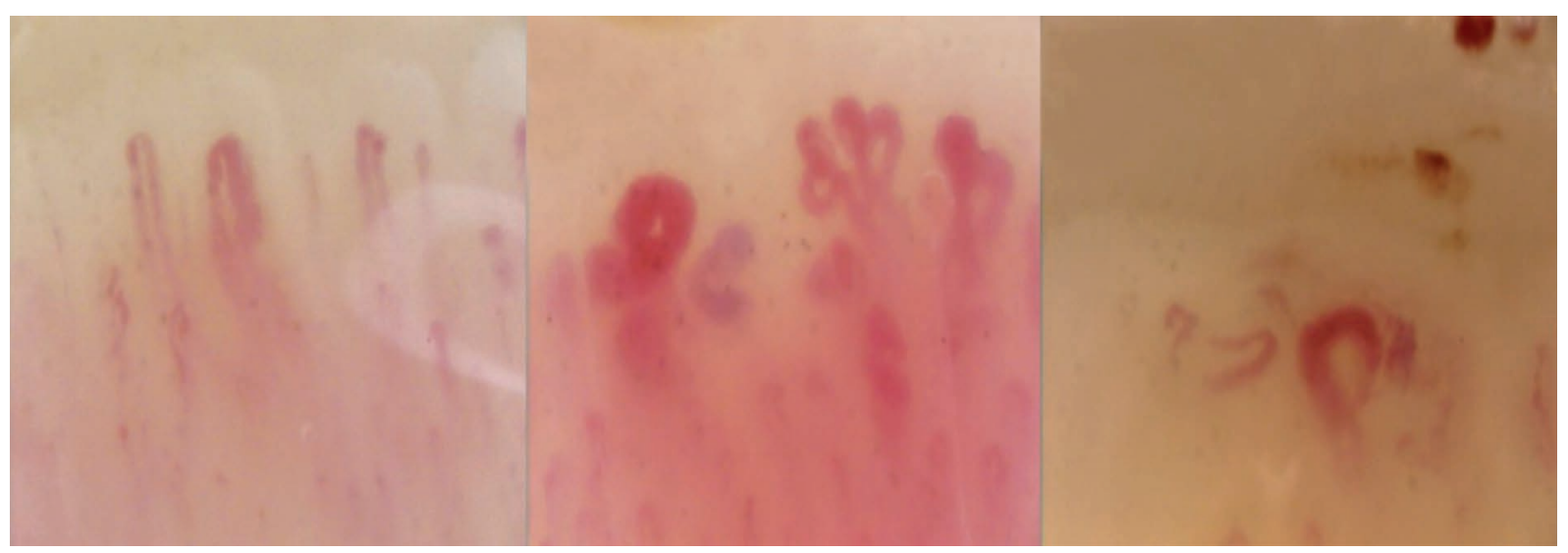

Figure 2. Scleroderma pattern: early, active and late. 
tients with scleroderma with a specificity of $81.4 \%$, a sensitivity of $92.3 \%$ and a predictive value of $84.3 \%$. The parameters used are: the presence of megacapillaries, the number and diameter of capillaries, the presence of arborescent capillaries and the neoangiogenesis. There are two problems: i) the absence of megacapillaries does not allow a proper risk stratification; ii) an increased estimate of false positives.

A subsequent application of the score CSURI allowed a correlation of the framework capillaroscopic as a prognostic factor upgradeability of complications in scleroderma. ${ }^{17}$

In addition, in the past years, some authors observed, in SSc patients, an association between reduction of the capillary density, and the severity of pulmonary arterial hypertension (PAH) (but not with the capillary loop dimension), suggesting that either systemic microvascular changes play a part in the development of PAH or PAH itself contributes to the systemic microvascular changes. Recently, Riccieri et al. ${ }^{18}$ observed in 12 consecutive SSc patients, all complicated by PAH, a significant correlation between pulmonary pressure values detected by right heart catheterization and the NVC modifications. In contrast, a low prevalence of NVC changes is described in idiopathic PAH compared with SSc patients, substantially consistent with those of healthy subjects. ${ }^{19,20}$

Kayser et al. ${ }^{18}$ observed that the avascular score significantly correlated with the survival of SSc patients and the scleroderma patients with higher degree of capillary loss (avascular score above 1.5) had a 2fold increase in mortality rates in comparison to those with a reduced amount of avascular score. ${ }^{21}$

Other two studies evaluated the association of capillaroscopic parameters and mortality with SSc and showed that an active pattern, according to the classification of Maricq and a loose of capillaries on capillaroscopy, was associated with shorter survival. ${ }^{22,23}$

Sebastiani et al. recently observed that presumably, capillaroscopy alone is unable to fully explore the complex pathophysiology of scleroderma microangiopathy, and other clinical, demographic, or serological parameters could be essential in the characterization of prognostic value of the microvascular damage. Therefore, the evaluation of large scleroderma population and more complex predictive model, including capillaroscopy and other clinical/serological variables, could reveal new significant associations with SSc manifestations and prognosis. ${ }^{24}$

Recent data show in scleroderma patients a strength correlation between angiogenic and angiostatic levels factors (as vascular endothelial growth factor, angiopoietin 2, endothelin-1, endostatin) and microangiopathy assessed by capillaroscopy.

On the other hand an association between capil- laroscopic pattern, and antibody was demonstrated in SSc. In fact antiendothelium antibodies serum levels were recently found to be higher in patients with the late NVC pattern compared with the early and active, and the prevalence of anti-Scl70 antibodies is significantly higher in the active and late versus the early patterns, whereas the prevalence of ACA was highest in patients with the early NVC pattern. ${ }^{15}$

\section{Monitoring under therapies}

Capillaroscopy may be useful to monitor non-invasively the remodeling of the microcirculation in various therapies and thus to assess their effectiveness. Some data on the effect of neo-angiogenic therapies with prostanoids have been widely documented, nevertheless conducted on limited case studies ${ }^{25-27}$ (Figure 3). Similarly, studies have detected a modification of capillaroscopic pattern under combination of endothelin inhibitors with prostanoids showing a neoangiogenetic greater effect of the combination of the two drugs, rather than the administration of a single drug. ${ }^{28}$

Changes in the capillaroscopic pattern with improvement of some regressive parameters (capillary density, flou effect, blood flow) were seen in only few isolated cases of patients with RF-I phenomenon in the course of treatment with aminaftone and other rheology drugs. ${ }^{29}$

\section{Nailfold capillaroscopy pattern: scleroderma spectrum}

The term scleroderma spectrum diseases includes connective tissue diseases characterized by microvascular alterations and often clinically associated with RP and sometimes specific antibodies. The most important are dermatomyositis/polymyositis (DM/PM), mixed connective tissue (MTCD), connective tissue undifferentiated syndromes overlap (UTCD). The main anomalies are those already known capillaroscopy scleroderma, often with different manifestation.

\section{Mixed connective tissue disease}

MTCD is characterized by various clinical manifestations and by the presence of the antibody ENA U1RNP; capillaroscopic findings are frankly pathological due to a scleroderma pattern active or advanced up to $60 \%$ of cases. The most frequent anomalies are the presence of capillary ectasias winding loops and bushy capillars expression of angiogenesis, in $50 \%$ of cases there is also a fair number of megacapillaries and avascular areas. These signs seem to have a predictive role in the development of $\mathrm{PAH}^{30}$ (Figure 4). 


\section{Undifferentiated connective tissue disease/overlaps syndromes}

In UTCD capillaroscopy is definitely a useful tool to discern a possible upgradeability to paintings more consistent with autoimmune diseases. In $40 \%$ of patients there are non-specific abnormalities especially morphological abnormalities with capillary ectasia and tortuosity. In $20 \%$ of them are present alterations consisting in the desertification of the comb, ectasia, megacapillaries, bleeding compatible with a scleroderma spectrum and which could also be suggestive of an evolution of the disease in scleroderma. ${ }^{31}$

\section{Dermatomyositis/polymiositis}

A defined pattern has been reported in patients affected by DM/PM. This pattern, often associated with aspects of the SSc pattern, includes the presence of two or more of the following findings in at least two nailfolds: enlargement of capillary loops, loss of capillaries, disorganization of the normal distribution of capillaries, budding (bushy) capillaries, twisted enlarged capillaries and capillary hemorrhages (extravasates); the alterations are often not present in all fingers. These aspects can mutate often in few days to respond to therapy. The persistence of nailfold alterations is a sign of poor prognosis ${ }^{32,33}$ (Figure 5).

\section{Capillaroscopy in autoimmune non scleroderma spectrum diseases}

\section{Systemic lupus erythematosus}

The framework capillaroscopic in systemic lupus erythematosus (SLE) is generally normal, in $30 \%$ of cases it is possible to observe abnormalities in non-specific load of capillary loops, venular visibility and sludging of bloodwidth variability of capillary loop length. A nailfold scleroderma pattern with enlarged loops and avascular areas is more frequent in SLE patients in association to anti-U1 ribonucleoproteins (RNP) and anti-cardiolipin (aCL) antibodies, while in association to antiphospholipids syndrome are frequent blackberries apical hemorrhages. ${ }^{30}$

\section{Antiphospholipids syndrome}

One study reported symmetrical microhemorrhages at nailfold analysis, which were found particularly significant in patients showing the presence of both serum immunoglobulin (Ig) $\mathrm{G}$ and IgM aCL but these changes could not be correlated to the impairment of functional parameters ${ }^{34-37}$ (Appendix Figure 1).

\section{Sjögren's syndrome}

Capillaroscopic abnormalities in Sjögren's syn-

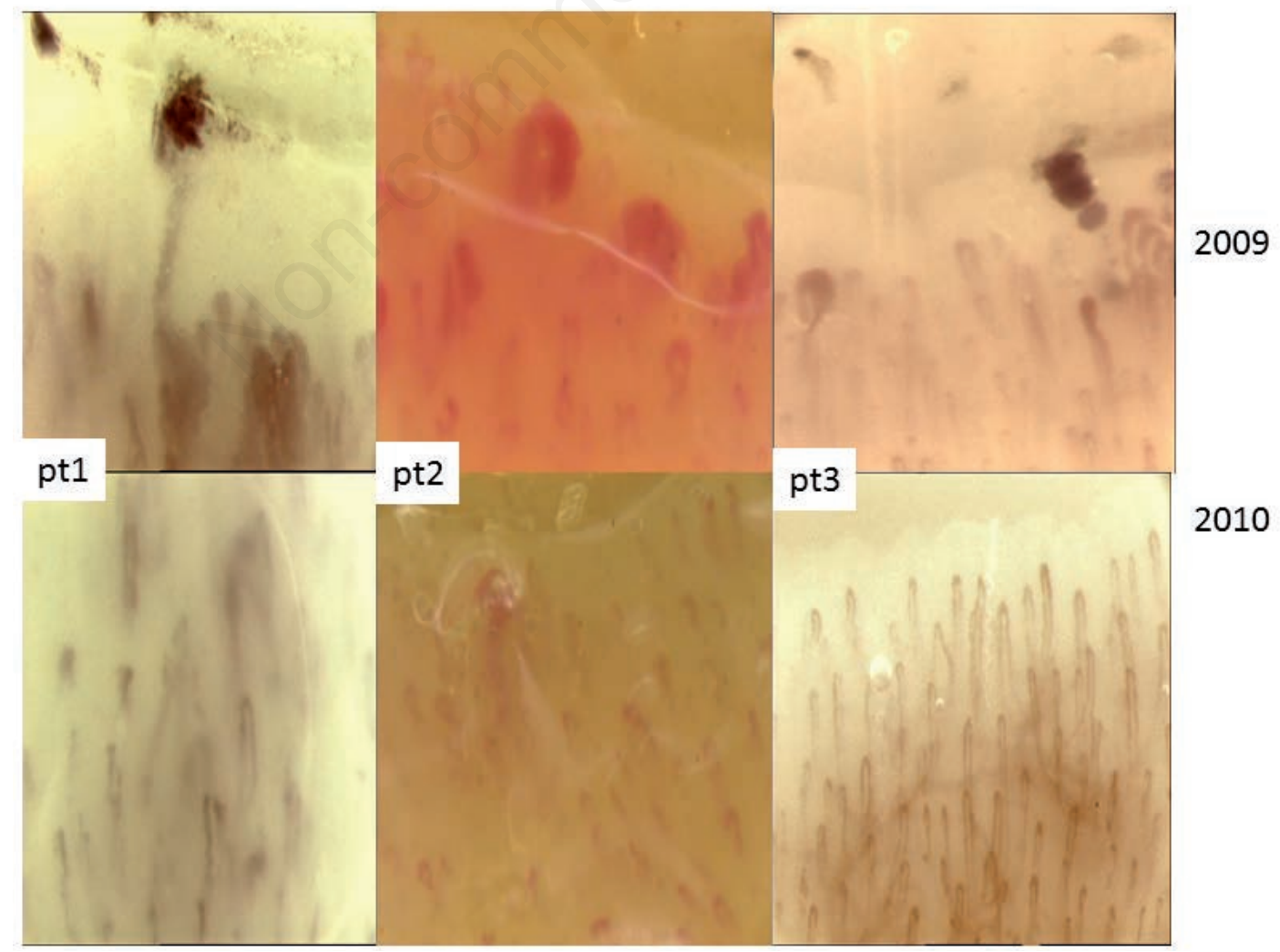

Figure 3. Neoangiogenetic effects of iloprost (Faggioli, personal data). 
drome (SS) ranged from non-specific findings (crossed capillaries) to more specific findings (confluent hemorrhages and pericapillary hemorrhages) or SSc-type findings. SS patients with RP presented capillary abnormalities in higher frequency than patients without RP. The majority of SS patients with ACA ( $80 \%)$ presented SSc-type findings ${ }^{38}$ (Appendix Figure 2).

\section{Rheumatoid arthritis}

The capillaroscopy pattern in patients with rheumatoid arthritis (RA) does not demonstrate specific alterations in the microcirculation, and many times the findings are similar to the normal subject. It was reported in some cases the presence of thin palesade loops and elongated with the venous plexus subpapillar, which is particularly visible and congested. This pattern is present in about $40 \%$ of cases and appears above all aspecific, furthermore according to a few authors it is correlated to the steroid therapy which is often present in about the totality of the patients $^{39,40}$ (Appendix Figure 3).

\section{Psoriatic arthritis}

The capillaroscopy in psoriatic arthritis patients, is often aspecific, but sometimes a reduction of capillary density is present as result of abhorrent neoangiognenesis. On the other hand there are more frequently microhemorrhages, increased flou effect, small loops and more bushy loops as expression of aberrant neoangiogenesis. These findings are present also in the cutaneous psoriatic plaques where often an increased density of capillaries ${ }^{40}$ is present (Appendix Figure 4).

\section{Capillaroscopy in internal medicine}

\section{Primary biliary cirrhosis}

Primary biliary cirrhosis (PBC) is frequently asso-

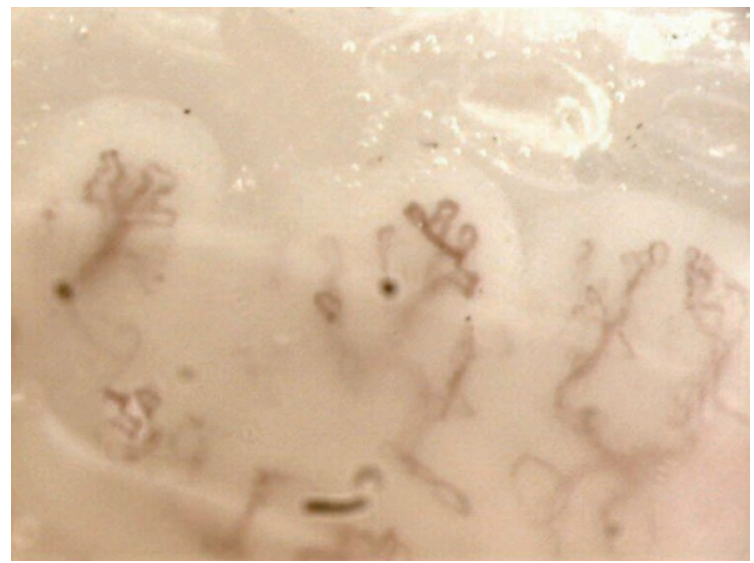

Figure 4. Capillaroscopic pattern in mixed connective tissue. ciated with other autoimmune diseases, including SSc which represents the most frequent autoimmune rheumatic disease associated with $\mathrm{PBC}$, with a prevalence ranging from $5 \%$ to $12 \%{ }^{41,42}$ (Appendix Figure 5).

In a large cohort of $\mathrm{PBC}$ patients, $12.5 \%$ satisfied diagnostic criteria for early SSc and $6.3 \%$ had definite $\mathrm{SSc}^{43}$

In a recent study more $\mathrm{PBC}$ patients with abnormal capillaroscopy findings were significantly positive for anticentromere antibody than those with normal capillaroscopy findings.

The association between PBC, ACA and antimitochondrial antibodies in overlap PBC/SSc led some authors to suggest a diagnostic algorithm in $\mathrm{PBC}$ in the presence of naifold capillaroscopy. ${ }^{44}$

\section{Buerger's disease}

Thromboangiitis obliterans (or Buerger's disease) is a rare inflammatory vasculitis that commonly involves small and medium-sized arteries of the extremities of tobacco smokers between the ages of 25 and 50. Although the diagnosis is based on the clinical picture and angiographic findings, the capillaroscopic findings show the presence of microhemorrhages tortuosity, edema, derangement of capillaries, arrest of capillary blood flow, and isolated mega-capillaries similar to alteration seen in scleroderma pattern disease spectrum, therefore nailfold capillaroscopy could be a useful tool to evaluate disease progression and the response to treatment. ${ }^{45}$

\section{Vasculitis}

There are few studies on capillaroscopy in vasculitis, Ander et al., studied 12 patients with granulomatosis with polyangiitis (GPA) and found capillaroscopic alterations in $92 \%$ of them with avascular areas being the characteristic finding in these patients. Capillary dilatations were not observed. ${ }^{46}$

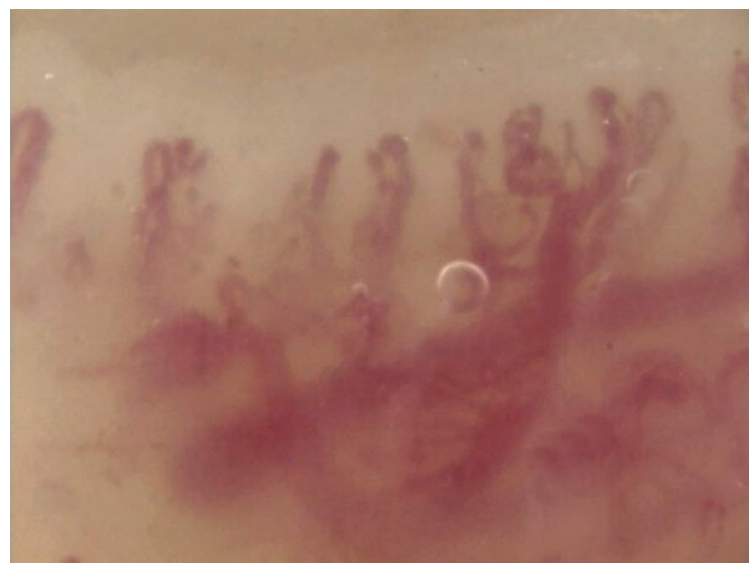

Figure 5. Capillaroscopic pattern in dermatomyositis/ polymiositis. 
In other series, we observed a higher frequency of twisting capillaries, increased bleeding, decreased capillary density and presence of avascular areas. None of these findings in periungueal capillaroscopy could be related to specific organ system involvement and this leads us to believe that patients with GPA present alterations in skin microcirculation. ${ }^{47}$

A prospective study of the capillaroscopy changes in 15 patients afflicted with various vasculitis periungual capillaroscopy showed isolated changes in 73\% of them. There were more changes in active disease but there no correlation was observed with generalized affliction (nervous system, kidneys and/ or skin). ${ }^{48}$

\section{Behçet's disease}

Enlarged capillaries were reported to be a common finding in patients with Behçet's disease in association with microhemorrhages and avascular areas, but none of them presents a statistical correlation with clinical manifestations. ${ }^{49}$

\section{Mixed cyroglobulinemia}

Until now pattern capillaroscopic specific to vasculitides has never been characterized. There are some reports, not reconfirmed, on morphological abnormalities and further slowing down of the flow in patients with cryoglobulinemia, despite the Raynaud's phenomenon is absent ${ }^{50}$ (Appendix Figure 6).

\section{Miscellaneous}

\section{Radioexposure}

In radioexposure $\mathrm{NVC}$ shows slight alterations of the microvascular blood flow and terminal blood. Aspecific findings in vessels network in the radiologists were found in reference to their length of service. ${ }^{51}$

\section{Hypertension}

Capillaroscopy has also been applied to assessing endothelial function by measuring skin capillary density and recruitment and also red blood cell velocity changes with post-occlusive reactive hyperaemia and this approach has been reported in testing numerous anti-hypertensive treatment. On the other hand a significant reduction in capillary density is well known to be present in hypertensive patients. ${ }^{52,53}$

Recently in the coronary slow flow phenomenon (CSFP) (delayed opacification of coronary arteries in the absence of significant stenosis) many nailfold capillary abnormalities including dilatation, tortuosity and microhemorrhages were observed in about $60 \%$ of patients suggesting the presence of inflammation and anatomical anomalies that can lead to CSFP. ${ }^{54}$

\section{Diabetes}

Systematic vasculopathy is the most common complication of diabetes mellitus (DM) and much of its morbidity is attributable to the macrovascular and microvascular complications related to the degree of hyperglycemia and hypertension. Reported changes in nailfold capillary morphology in DM include microaneurysms, apical dilatations, branching and hemorrhagic extravasations. However these features, with the exception of microaneurysms, can also be found in healthy subjects. A high frequency of dilatation at the apex of loops may be the key feature in diabetes, but these changes do not appear to be linked to disease duration or disease sub-type (Appendix Figure 7).

Morphological and functional changes in capillary microscopy have been reported in diabetic patients with type $1 \mathrm{DM}$.

In DM 1 at NVC was reported spiral loops with decreased density and capillary dilatation in $59 \%$ of patients with microangiopathy versus only $13 \%$ in diabetic patients without microangiopathy, suggesting the possibility of using NVC in monitoring the severity of vascular changes.

In addition, other authors have reported the correlation between microvascular changes in capillaroscopy and the occurrence of retinopathy and plasma level of selectin and IL18 in 25 patients with type 1 DM. ${ }^{55}$

\section{Critical limb ischemia}

The vessels of the microcirculation are not routinely assessed in clinical practice in the management of critical limb ischemia.

In patients with mild disease, the capillary bed of the forefoot does not seem to be markedly abnormal, however, with the advent of moderate or severe occlusion profound abnormalities of the skin capillaries have been reported. Fagrell and Lundberg reported a classification system for foot capillaries which was predictive for the development of necrosis and was much more sensitive than systolic blood pressure at the toes. However it has enabled the mapping of a foot to indicate areas at risk ${ }^{56-58}$ (Appendix Figure 8).

\section{Oral capillaroscopy}

Another area where the capillaries become visible is the oral mucous, this additional facility of capillaroscopy is useful if the digital site is not practicable (e.g., ulcers, amputations, etc.). Several studies have been made in this regard comparing the two methods, which differ substantially for the different orientation of the capillary comb (parallel rather than perpendicular to the surface displayed). The use in rheumatology has allowed us to confirm aspects of the pattern 
visible at nailfold areas (RA, Sjögren syndrome, SSc), and has identified some specific frameworks that do not correspond to nailfold findings as in Hashimoto's thyroiditis, oral lichen planus, burning mouth syndrome $^{59}$ (Appendix Figure 9).

\section{Current status of capillaroscopy and Conclusions}

As of today 398 articles concerning the use of capillaroscopy in rheumatic diseases and internal medicine have been published on pubmed. Almost $97 \%$ of these articles agree in the statement that the capillaroscopy is one of the gold standard methods for noninvasive examination of the microcirculation and it plays an important role in screening, monitoring and prognosis of systemic sclerosis.

Several ongoing studies are stressing the diagnostic role of capillaroscopy also in many other diseases with the characterization of specific patterns.

\section{References}

1. De Martinis M, Ginaldi L. Capillaroscopy opens a window to look inside. Rheumatol Curr Res 2014;4:1.

2. Maricq HR, LeRoy EC. Patterns of finger capillary abnormalities in connective tissue disease by 'wide-field' microscopy. Arthritis Rheum 1973;16:619-28.

3. Jung P, Trautinger F. Capillaroscopy. JDDG 2013;11: 731-6.

4. Mazzotti G, Bredemeier M, Breno CV, et al. Assessment of nailfold capillaroscopy in systemic sclerosis by different optical magnification methods. Clin Exp Dermatol 2014;39:135-41.

5. Cutolo M, Sulli A, Smith V. How to perform and interpret capillaroscopy. Best Pract Res Clin Rheumatol 2013 ;27:237-48.

6. Allen J, Howell K. Microvascular imaging: techniques and opportunities for clinical physiological measurements. Physiol Meas 2014;35:R91-141.

7. Batticiotto A, Atzeni F, Folia S, et al. Feet nailfold capillaroscopy is not useful to detect the typical scleroderma pattern. Clin Exp Rheumatol 2012;30:S116-7.

8. Wildt M, Wuttge DM, Hesselstrand R, Scheja A. Assessment of capillary density in systemic sclerosis with three different capillaroscopic methods. Clin Exp Rheumatol 2012;30:S50-4.

9. Sulli A, Secchi EM, Pizzorni C, Cutolo M. Scoring the nailfold microvascular changes during the capillaroscopic analysis in systemic sclerosis patients. Ann Rheum Dis 2008;67:885-7.

10. Ingenioli F, Gualtierotti R, Lubatti C, et al. Nailfold capillary patterns in healthy subjects: a real issue in capillaroscopy. Microvasc Res 2013;90:90-5.

11. Nagy Z, Czirjàk L. Nailfold digital capillaroscopy in 447 patients with connective tissue disease and Raynaud's disease. J Eur Acad Dermatol Venereol 2004;18:62-8.

12. Cutolo M, Sulli A, Pizzorni C, Accardo S. Nailfold videocapillaroscopy assessment of microvascular damage in systemic sclerosis. J Rheumatol 2000;27:155-60.
13. Ingegnoli $F$, Boracchi $P$, Gualtierotti $R$, et al. Improving outcome prediction of systemic sclerosis from isolated Raynaud's phenomenon: role of autoantibodies and nailfold capillaroscopy. Rheumatology 2010;49:797-805.

14. Jung P, Trautinger F. Capillaroscopy. J Dtsch Dermatol Ges 2013;11:731-6.

15. Cutolo M, Smith V. State of the Art on nailfold capillaroscopy. Rheumatology 2013,52:1933-40.

16. Ingegnoli F, Boracchi P, Gualtierotti R, et al. Prognostic model based on nailfold capillaroscopy for Raynaud's phenomenon. Identifying patients at high risk for the development of a scleroderma spectrum disorder: PRINCE. Arthrit Rheum 2008;58:2174-82.

17. Sebastiani M, Manfredi A, Lo Monaco A, et al. Capillaroscopic skin ulcers risk index (CSURI) calculated with different videocapillaroscopy devices: how its predictive values change. Clin Exp Rheumatol 2013;31:S115-7.

18. Riccieri V, Vasile M, Iannace N, et al. Systemic sclerosis patients with and without pulmonary arterial hypertension: a nailfold capillarocopy study. Rheumatology 2013; 52:1525-8.

19. Ohtsuka T, Hasegawa A, Nakano A, et al. Nailfold capillary abnormality and pulmonary hypertension in systemic sclerosis. Int J Dermatol 1997;36:116-22.

20. Hofstee HM, Vonk Noordegraaf A, Voskuyl AE, et al. Nailfold capillary density is associated with the presence and severity of pulmonary arterial hypertension in systemic sclerosis. Ann Rheum Dis 2009;68:191-5.

21. Kayser C, Sekiyama JY, Pròspero LC, et al. Nailfold capillroscopy abnormalities as predictors of mortality in patients with systemic sclerosis. Clin Exp Rheumatol 2013;31:103-8.

22. Lee P, Leung FY, Alderdice C, Armstrong SK. Nailfold capillary microscopy in the connective tissue diseases: a semiquantitative assessment. J Rheumatol 1983;10:930-8.

23. Scussel-Lonzetti L, Joyal F, Raynauld JP, et al. Predicting mortality in systemic sclerosis: analysis of a cohort of 309 French Canadian patients with emphasis on features at diagnosis as predictive factors for survival. Medicine 2002;81:154-67.

24. Sebastiani M, Manfredi A, Cassone G, et al. Measuring microangiopathy abnormalities in systemic sclerosis patients: the role of capillaroscopy-based scoring models. Am J Med Sci 2014;348:331-6.

25. Faggioli P, Giani L, Mazzone A. A possible role of iloprost (stable analog of PGI2) in promoting neoangiogenesis in systemic sclerosis. Clin Exp Rheumatol 2006;24:220-1.

26. Shah P, Murray AK, Moore TI, Herrick AI. Effects of iloprost on microvascular structure assessed by nailfold videocapillaroscopy: a pilot study. Rheumatol 2011;38: 2079-80.

27. Wigley FM, Wise RA, Seibold JR, et al. Intravenous iloprost infusion in patients with Raynaud's phenomenon secondary to systemic sclerosis. A multicenter, placebocontrolled, double-blind study. Ann intern Med 1994; 120:199-206.

28. Cutolo M, Zampogna G, Vremis L, et al. Longterm effects of endothelin receptor antagonism on microvascular damage evaluated by nailfold capillaroscopic analysis in systemic sclerosis. J Rheumatol 2013;40:40-5.

29. Faggioli P, Sciascera A, Gilardi AG, et al. Aminaftone: a possibile role in the management of Raynaud's phenomenon. Ital J Med 2014;8(Suppl 2):42. 
30. Furtado RN, Puccinelli MI, Cristo VV, et al. Scleroderma-like nailfold capillaroscopic abnormalities are associated with anti U1-RNP antibodies and Raynaud's phenomenon in SLE patients. Lupus 2002;11:35-41.

31. Mariq HR, Valter I. A working classification of scleroderma spectrum disorders: a proposal and the results of testing on a sample of patinets. Clin Exp Rheumatol 2004; 22:S5-13.

32. Klyscz T, Bogenschutz O, Junger M, Rassner G. Microangiopathic changes and functional disorders of nailfold capillaries in dermatomyositis. Hautarzt 1996;74: 289-93.

33. Selva-O'Callaghan A, Fonollosa-Pla V, TralleroAraguas E, et al. Nailfold capillary microscopy in adults with inflammatory myopathy. Sem Arthritis Rheum 2010;39:398-404.

34. Bongard O, Bounameaux H, Miescher PA, De Moerloose P. Association of anticardiolipin antibodies and abnormal nailfold capillaroscopy in patients with systemic lupus. Lupus 1995;4:14-21.

35. Sulli A, Pizzorni C, Cutolo M. Nailfold videocapillaroscopy abnormalities in patients with antiphospholipid antibodies. J Rheumatol 2000;27:1574-6.

36. Vaz JL, Dancour MA, Bottino DA, Bouskela E. Nailfold videocapillaroscopy in primary antiphospholid syndrome (PAPS). Rheumatology 2004;43:1025-7.

37. Cutolo M, Sulli A, Secchi E, et al. Nailfold capillaroscopy is useful for the diagnosis and follow up of autoimmune rheumatic diseases. A future tool for the analysis of microvascular heart involvement? Rheumatology 2006;54:43-6.

38. Capobianco KG, Xavier RM, Bredmeier M, et al. Nailfold capillaroscopic findings in primary Sjogrens syndrome: clinical and serological correlations. Clin Exp Rheumatol 2005;23:789-94.

39. Kabasakal Y, Elvins DM, Ring EF, Mc Hugh NJ. Quantitative nailfold capillaroscopy findings in a population with connective tissue disease and in normal heathy controls. Ann Rheum Dis 1996;55:507-12.

40. Graceffa D, Amorosi B, Maiani E, et al. Capillaroscopy in psoriatic and rheumatoid arthritis: useful tool for differential diagnosis. Arthritis 2013;2013:957480.

41. Marasini B, Gagetta M, Rossi V, Ferrari P. Rheumatic disorders and primary biliary cirrhosis: an appraisal of 170 Italian patients. Ann Rheum Dis 2001;60:1046-9.

42. Watt FE, James OF, Jones DE. Patterns of autoimmunity in primary biliary cirrhosis patients and their families: a population-based cohort study. QJM 2004;97:397-406.

43. Tovoli F, Granito A, Giampaolo L, et al. Nailfold capillaroscopy in primary biliary cirrhosis: a useful tool for the early diagnosis of scleroderma. J Gastrointestin Liver Dis 2014;23:39-43.

44. Liberal R, Grant CR, Sakkas L, et al. Diagnostic and clinical significance of anti-centromere. Clin Res Hepatol Gastroenterol 2013;37:572-85.
45. Guidelli GM, Bardelli M, Fioravanti A, Selvi E. Capillaroscopic abnormalities in Buerger's disease. Eur J Rheumatol 2014;1:81-3.

46. Anders HJ, Headecke C, Sigl T, Kruger K. Avascular areas on nailfold capillary microscopy of patients with Wegener's granulomatosis. Clin Rheumatol 2000;19:86-8.

47. Uceda Montanes J, Martìnes Pérez R, Velloso Feijoo ML, Marenco de la Fuente J. Periungueal capillaroscopy findings in a series of patients with granulomatosis with polyangiitis (Wegener): an observational study. Open J Rheumatol Autoimmune Dis 2013;3:130-4.

48. Sendino Revuelta A, Barbado Hernàndez FJ, Torrijos Eslava A, et al. Capillaroscopy in vasculitis. Ann Med Intern 1991;8:217-20.

49. Aytekin S, Yuksel EP, Aydin F, et al. Nailfold capillaroscopy in Behcet disease, performed using videodermoscopy. Clin Exp Dermatol 2014;39:443-7.

50. Wysenbeek AJ, Leibovici L, Cohen S, Weinberger A. Nailfold capillary microscopy in essential mixed cryoglobulinemia: a report of several cases. Angiology 1985; 36:552-5

51. Pennarola R, Perderau B, Trenta G, Cosset JM. Nailfold capillroscopic monitoring as preventive medicine in subjects exposed to ionising radiation. Madrid: IRPA Publications; 2004.

52. Kaiser SE, Sanjuliani AF, Estato V, et al. Hypertensive treatment improves microvascular rarefaction and reactivity in low-risk hypertensive individuals. Microcirculation 2013;20:703-16.

53. Gasser P, Buhler FR. Nailfold microcirculation in normotensive and essential hypertensive subjects, as assessed by video-micoscopy. J Hypertens 1992;10:83-6.

54. Yuksel S, Pancar Yuksel E, Yenercag M, et al. Abnormal nailfold capillaroscopic findings in patients with coronary slow flow phenomenon. Int J Clin Exp Med 2014;7:1052-8.

55. Kuryliszyn-Moskal A, Dubicki A, Zarzycki W, et al. Microvascular abnormalities in capillaroscopy correlate with higehre serum IL-18 and sE-selectin levels in patients with type 1diabetes complicated by microangiopathy. Folia Histochem Cytobiol 2011,49:104-10.

56. Kluz J, Malecki R, Adamiec R. Pratical importance and modern methods of the evaluation of skin microcirculation during chronic lower limb ischemia in patients with peripheral arterial occlusive disease and/or diabetes. Intern Angiol 2013;32:42-51.

57. Fagrell B, Lundberg G. A simplified evaluation of vital capillary microscopy for predicting skin viability in patients with severs arterial insufficiency. Clin Physiol 1984;4:403-11.

58. Shore AC. Capillaroscopy and the measurement of capillary pressure. Br J Clin Pharmacol 2000;50:501-13.

59. Scardina GA, Ruggeri A, Messina P. Oral microcirculation observed in vivo by videocapillaroscopy: a review. J Oral Sci 2009;51:1-10. 